\title{
Self-Limited Accumulation of Colloids in Porous Media
}

\author{
G. Gerber ${ }^{1,2}$ M. Bensouda, ${ }^{1}$ D. A. Weitz, ${ }^{2}$ and P. Coussot ${ }^{1}$ \\ ${ }^{1}$ Université Paris-Est, Laboratoire Navier (ENPC-IFSTTAR-CNRS), Champs-sur-Marne 77420, France \\ ${ }^{2}$ Experimental Soft Condensed Matter Group, School of Engineering and Applied Sciences, Harvard University, \\ Cambridge, Massachusetts 02138, USA
}

(Received 10 April 2019)

\begin{abstract}
We present local direct imaging of the progressive adsorption of colloidal particles inside a 3D model porous medium. By varying the interparticle electrostatic interactions, we observe a large range of particle deposition regimes, from a single layer of particles at the surface of the medium to multiple layers and eventually clogging of the system. We derive the complete deposition dynamics and show that colloid accumulation is a self-limited mechanism towards a deposited fraction associated with a balance between the particle interactions and the imposed flow rate. These trends are explained and predicted using a simple probability model considering the particle adsorption energy and the variation of the drag energy with evolving porosity. This constitutes a direct validation of speculated particle transport mechanisms, and a further understanding of accumulation mechanisms.
\end{abstract}

DOI:

Colloidal particles from industrial or natural sources propagate and alter the environment they flow through. Common problems include the accumulation of particles impacting industrial [1,2] or biological [3-6] processes (filtration, storage, cleaning, sorting, etc.) or the leaking of contaminants in ground water [7-9]. Predicting particle transport and stoppage in these porous media is key to solve these problems. All stoppage events have either a geometrical (i.e., straining [10], clogging [11], bridging [12]) or a physicochemical origin (i.e., adsorption). Although the elementary processes are well identified, their development in a porous medium is often described by empirical models. Moreover, because of the complexity of real 3D porous structures, the dynamics of particle deposition are usually extrapolated from indirect observations like breakthrough curves [13-15], direct observations in simplified systems (2D and/or at pore scale) [16-19], and qualitative static imaging or indirect imaging in realistic systems [20,21].

For attractive interactions between suspended particles and porous matrices, several regimes are speculated but unequally understood. First, a particle following a streamline close enough to a surface can be intercepted and adsorbed. Subsequent adsorption is then limited by the presence of previously adsorbed particles, and by interparticle repulsion [22]. This leads to the formation of a uniform single layer of adsorbed particles well described by a simple deposition model relying on a blocking function $[23,24]$ and the DLVO theory $[25,26]$. Then, for weak interparticle repulsion, particles can adsorb to each other and form complex deposits not limited to the surfaces. As deposits can then grow to larger sizes they may induce significant changes to the flow and boundary conditions, which makes the system much more difficult to study. These situations are generally ignored or described assuming no coupling between the deposition mechanisms and the state of the flow [27].

Here, we present a full description - by direct internal observation in a model system - of the successive states of deposition from the formation of a monolayer of particles to multilayer accumulation and potential clogging. We explore how the deposition dynamics are affected by the coupled evolution of the pore space and hydrodynamics. Finally, we show that the accumulation of particles can be a self-limited phenomenon driven by a competition between the drag force applied on the adsorbing particles and the colloidal interaction energy.

As a model porous medium we use monodisperse borosilicate beads (negatively charged, $63 \mu \mathrm{m}$ average diameter) randomly packed (porosity $\phi_{0}=0.38$ ) in a square glass capillary (width $w=1.0 \mathrm{~mm}$ ), and we invade this medium with positively charged, fluorescent, monodisperse (diameter $d_{p}=1.0 \mu \mathrm{m}$ ) latex colloids. The suspensions are made at constant dilute concentration $C_{0}=$ $20 \mathrm{mg} \mathrm{mL}^{-1}$ in a mix of deionized water and DMSO to match the refractive index of the beads [28]. This allows us to visualize the particles inside the porous medium by confocal microscopy. The opposite charges of the beads and the particles fosters particle adsorption at the surface of the beads. Particle-particle bonding may be promoted by screening the interparticle repulsion (i.e., decreasing the Debye length) through an increase of the ionic strength $I$ by addition of salt (sodium iodide) [29].

A test consists of continuously injecting a suspension in the porous medium (initially saturated with liquid) and tracking the particle deposition inside the medium. Except when mentioned, we impose a constant flow rate 
$Q_{0}=5 \mu \mathrm{L} \mathrm{min}^{-1}$, which implies that the local mean velocity, i.e., $v_{0}=Q_{0} / w^{2} \phi_{0}$, increases when the porosity decreases. The flow is laminar: the Reynolds number at the maximum flow rate used in this study is $\operatorname{Re}=v_{0} \rho l / \eta=1.4 \times 10^{-3} \ll 1$, with $l=15 \mu \mathrm{m}$ the typical pore scale, $\eta=2.5 \mathrm{mPa}$ and $\rho=1.1 \mathrm{~kg} \mathrm{~m}^{-3}$ the viscosity and density of the mix. At some distance from the entrance, the concentration of suspended particles may significantly vary as a function of the history of adsorption all along the porous medium. Here we focus on the processes occurring around the entrance of the medium (i.e., over a distance of a few bead diameters), where the impact of adsorption history is expected to be negligible, so that the concentration of suspended particles is considered constant and equal to the injected concentration [29].

Let us first focus on negligible interparticle bonding due to strong repulsive electrostatic interactions (i.e., $\left.I=10^{-6} \mathrm{M}\right)$. Over time, the beads are progressively covered by particles adsorbed to their surface (see Fig. $\left.1, I_{1}-t_{2}\right)$. A steady state is reached after $1 \mathrm{~h}$ of injection and characterized by a single layer of particles, outlining the surface of the beads (see Fig. $1, I_{1}-t_{3}$ and $t_{4}$ ). The coverage is uneven, with a fairly uniform, significant coverage on the upstream side of the beads but a negligible one on the downstream side. For a single bead in a uniform, laminar, fluid flow (far from the bead), the streamlines are symmetrical [30] with regards to a cross section. Extrapolating this situation to the flow around packed beads on average we deduce that a particle following a streamline getting closer from the surface than its own radius will theoretically first intercept the surface of

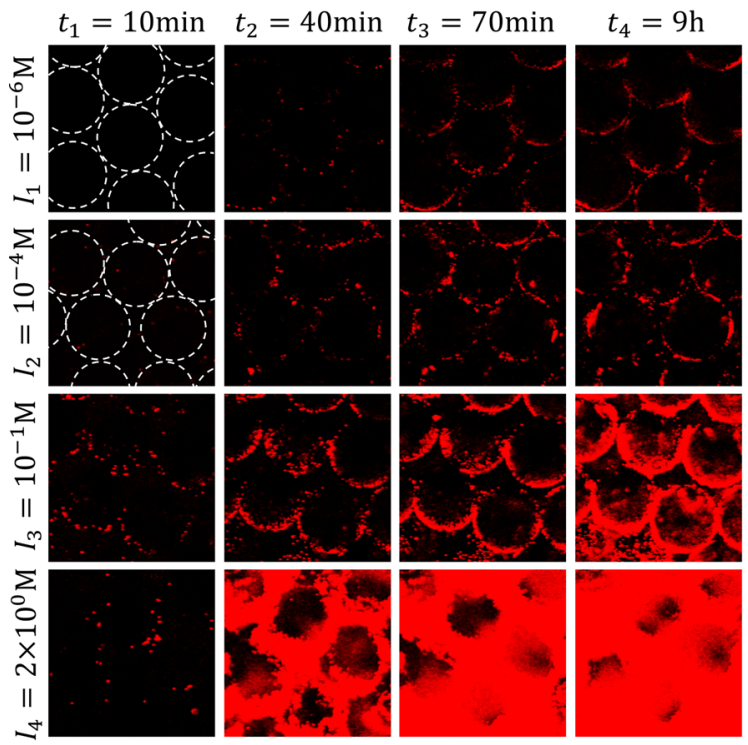

FIG. 1. Confocal microscopy images of particles (red) deposited in the pore space between glass beads (black) in a $150 \times 150 \mu \mathrm{m}^{2}$ window at the entrance of the porous media [see Fig. 2(c)], with the suspension flowing upwards. 4 conditions of ionic strength are presented at 4 times. the upstream bead face. The collision leads to a surface adsorption event, or a bouncing of the particle to a nearby streamline. This means than the observed preferential adsorption on the elements of surface facing the flow is related to the finite size of the particles.

We also observe that the trajectories of particles are rather smooth at relatively large distance from the walls but become more fluctuating at the approach of some wall [see Fig. 2(a)]. It is worth emphasizing that such effects do not result from inertia effects (small $\mathrm{Re}$ ) or from diffusion due to thermal agitation, as the Peclet number (i.e., $\mathrm{Pe}=d_{p} v_{0} / D$, with $D=10^{-13} \mathrm{~m}^{2} \mathrm{~s}^{-1}$ the diffusion coefficient of the particles) is over 100. Smooth trajectories would be obtained for a single particle moving through a simple bead packing [29]. This effect therefore likely results from boundary conditions continuously evolving due to other particles in suspension and depositions at the wall, which in particular rapidly changes the apparent bead roughness. A particle will be even more sensitive to these effects as it is closer to the wall, which explains these apparent fluctuations in the trajectories growing as the particles approach the wall. This likely enhances the adsorption of particles at the bead surfaces, as it temporarily places them closer to the walls. These fluctuations significantly affect the frequency of attempt of adsorption, which depends in a complex way on the particle concentration, the flow rate, and the current structure of the deposit. Here, as a critical aspect of our approach, we will consider this frequency as a constant factor for a
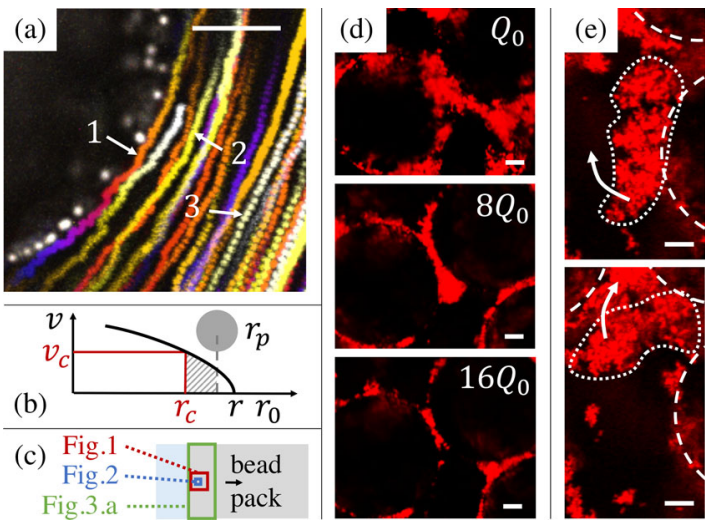

FIG. 2. Local transport and adsorption mechanisms. All scale bars are $10 \mu \mathrm{m}$. (a) Time projection of confocal imaging, with the suspension flowing upwards. $I=10^{-6} \mathrm{M}$. Steady particles appear as white dots, while moving particles are represented by their trajectories. Selected trajectories: particle 1 along wall, 2 close to wall, 3 far from wall. (b) Schematic velocity profile in a pore of initial and current radii $r_{0}$ and $r$. Dashed area shows positions favorable to adsorption: $\left[r_{c}: r-r_{p}\right]$. (c) Schematics of the three scales used in Figs. 1, 2 and 3(a). (d) Selected area under steady state for different flow rates $\left[Q_{0} ; 8 Q_{0} ; 16 Q_{0}\right]$, at $I=10^{-1} \mathrm{M}$. (e) Cluster formed on a surface at $I=10^{-1} \mathrm{M}$ (top), detaching and moving to a more stable position (bottom). Dashed lines highlight bead surfaces. 
given system, while, as described below, the probability of adsorption will significantly depend on the characteristics and evolution of the system. Such an approach relies on the same fundamental assumptions as the basic approach of adsorption [31] or the "Eyring model" for describing the viscosity of a simple liquid [32].

Then, if a particle encounters a free site, it can adsorb to it with some probability resulting from the surface-particle interactions and its motion characteristics. Otherwise, if it encounters an adsorbed colloid, it will be repulsed and will progress further along its streamline. A fundamental observation is our experiments is that no particle deposited on the beads detaches later. Thus, neglecting in first approximation the variation of boundary conditions due to progressive particle adsorption, the probability of adsorption depends on the flow rate, and is proportional to the particle concentration and to the fraction of bead surface still available for adsorption. Let us call $s$ the surface coverage, i.e., the ratio of the current number of particles adsorbed at the surface $S$ to the maximum possible value under our flow conditions $S_{0}$. Then, the rate of variation of $s$ writes as $\partial s / \partial t=k(1-s)$. In this expression $1-s$ is the available surface coverage, and $k$ is a factor including the frequency of attempt of adsorption and the probability of adsorption for a particle approaching a free wall. This model is a specific case of the Langmuir approach initially developed for molecular adsorption, but here without detachment. It solves as $s=1-\exp (-k t)$.

From the images, we compute the fluorescence $F$ over time and over a large number of beads, i.e., the whole capillary entrance [see Fig. 2(c) and [29] ]. With regards to a saturated surface deposition $S_{0}$, for $I=10^{-6} \mathrm{M}$, the deposition $F / S_{0}$ increases gradually before reaching a plateau value around 1 [see Fig. 3(a)] associated with a single layer of particles (see Fig. 1. $I_{1}-t_{4}$ ). This dynamics is well described by the above model for $s(t)$ [see Fig. 3(a)] with $k=5.0 \times 10^{-4} \mathrm{~s}^{-1}$, which confirms our understanding of the whole process of monolayer deposition.

Let us now see how the process evolves when interparticle bonding is allowed, by increasing the ionic strength. For a relatively low ionic strength (i.e., $I=10^{-4} \mathrm{M}$ ) we observe a change in the distribution of the deposits (see Fig. $1, I_{2}-t_{3}$ ): the process again starts by particle adsorption on the bead surface but it is soon followed by particle adsorption to other particles, which leads to the formation of deposits thicker than one particle size.

Under these conditions, since the particles keep the same properties all along the process, we would expect a continuous adsorption until filling the whole pore space and thus clogging of the system. On the contrary, we observe (see Fig. $1, I_{2}-t_{4}$ ) that the deposition progressively slows down and seems to tend to a maximum, significantly before saturation of the whole pore space. More precisely, the deposited amount increases at a decreasing rate and finally tends to a plateau at long times [see Fig. 3(a)]. Thus,
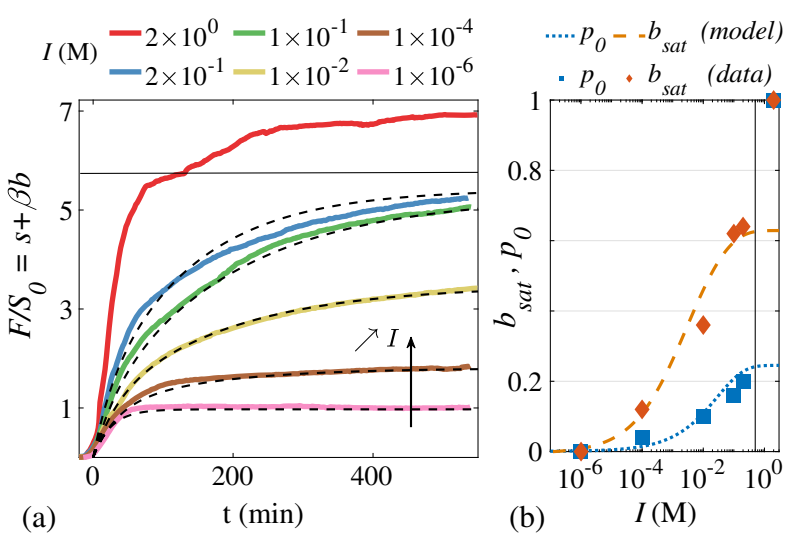

FIG. 3. (a) Overall deposition $F$ over time rescaled by surface saturation $S_{0}$, at the entrance of the porous media for increasing ionic strengths (bottom to top). Plain lines correspond to data. Values above the horizontal line are not quantitative due to resolution limitations (see Ref. [29]). Dashed lines show the fitted model: $s(t)$ for $I=10^{-6} \mathrm{M}$, with $k=5.0 \times 10^{-4} \mathrm{~s}^{-1} ; s(t)+\beta b(t)$ for larger values of $I$, with $\tau=3.0 \times 10^{4} \mathrm{~s}$. (b) Calculated and measured values for the initial bulk deposition probability $p_{0}$ (dotted line, squares) and the bulk deposition at saturation $b_{\text {sat }}$ (dashed line, diamonds), as a function of the ionic strength. The vertical line shows the limit of application of the model (i.e., suspension instability).

colloid deposition appears limited by some self-equilibrium process.

This implies that the flow evolution plays a major role: as the deposit thickness increases, the porosity decreases, and thus the local velocity increases (at constant flow rate); the drag force on the particles then increases, which decreases the probability of adsorption. Beyond some critical velocity the drag force is so large that no adsorption is possible.

This interpretation is confirmed by additional observations. By stepping the flow rate from $Q_{0}$ to $16 Q_{0}$, we obtain successive steady stationary deposits [see Fig. 2(d)]. The particles are washed from the large velocity regions and remain stable in the low velocity regions (i.e., around contact points), which provides a straightforward demonstration of the impact of velocity on the deposit thickness at equilibrium. At this point, it is also interesting to note that no detachment of particle is observed as long as deposition is far from its saturation value. This means that the velocity field variations do not affect the cohesion of individual particles. As we approach the stationary conditions though, during injection at a constant flow rate, some clusters of particles can suddenly detach and move slightly further in the medium [see Fig. 2(e)]. This illustrates that the local drag conditions, which initially allowed the formation of the deposit, have then evolved. The detachment of large clusters instead of individual particles likely results from the increase of the drag force on cohesive obstacles inducing a larger torque on the cluster.

Finally, with further screening of the repulsion (i.e., increasing $I$ ), the adsorption rate is larger, and the plateau 
of deposition increases [Fig. 3(a)]. We conclude that the particle deposition is essentially governed by some equilibrium between the flow conditions and the colloidal interactions. Such a qualitative conclusion matches standard theoretical analysis in that field [33], but we here have access to a detailed description of the process, from the local to the average level, which emphasizes the main trends of colloidal deposition (plateaux) and provides quantitative data for the local rate of adsorption in time. Note that with a strong screening of the interparticle repulsion $\left(I=2 \times 10^{-0} \mathrm{M}\right)$, heavy bulk deposition occurs at early times (Fig. 1, $I_{4}-t_{2}$ ). Even though this coincides with the limitation of our observation method due to massive diffraction (see Fig. $1, I_{4}-t_{3}$ and [29]), particles likely occupy a large fraction of the pore space. In that case, no self-equilibrium prevents the clogging of the system.

We can describe this process through a simple model. As a particle approaches a previously deposited particle, it interacts with it through Van der Waals forces and electrostatic forces. This interaction is well represented by a potential well $\Delta \Phi$ increasing with ionic strength, as the screening of the electrostatic repulsion increases (see Ref. [29] for a complete description of $\Delta \Phi$, function of the Hamaker constant $A_{H}$, and the particle surface potential $\left.\Phi_{0}\right)$. We can thus expect that while it gets sufficiently close to an already adsorbed particle, the former will tend to fall in this potential well. A particle will then remain attached if the drag force exerted by the liquid flow around it is sufficiently small. In other words, in order to remove this particle from its potential well, the liquid velocity around the particle must be such that the work $W_{d}=6 \pi d_{p}^{2} \eta v$ due to drag force during the particle displacement out of the well (thus over a typical distance of the order of the particle size), is larger than the depth of the well, i.e., if $W_{d}>\Delta \Phi$. The balance of these energies defines a critical fluid velocity $v_{c}$. For a flow through any geometry, the fluid velocity increases with the distance from the walls, which is confirmed by our observations (considering that, in general, moving particles have the same velocity as the fluid) [see Fig. 2(a)]. As a consequence, we can define a critical position $r_{c}$ at which $v\left(r_{c}\right)=v_{c}$, such that only the particles situated at a distance greater than $r_{c}$ from the pore axis may adsorb [see Fig. 2(b)]. On the other side, particles are sterically constrained at a distance (from particle center to wall) larger than one particle radius $r_{p}$. Assuming homogeneous particle dispersion in the fluid, the interval $\left[r_{c} ; r-r_{p}\right]$ compared to the full pore volume defines the fraction of particles in a place suitable for adsorption, thus proportional to a probability of adsorption $p$. For a given flow rate the exact critical values and adsorption probability depend on the detailed boundary conditions, i.e., the porous medium structure, which changes with the volume of deposited particles. On average (over a larger number of pores), we thus expect a probability $p$ depending on the fraction of pores occupied by deposited particles, i.e., $b$.
More precisely, $b$ is the ratio of the number of particles adsorbed to other particles $B$ to the maximum number of particles that fit in the pore space $B_{0}$, excluding the maximum number of particles in the surface layer $S_{0}$. Note that the ratio $\beta=B_{0} / S_{0}$ is a bulk to surface filling capacity ratio. Under these conditions the adsorption rate expresses as $\partial b / \partial t=p(b)$. Consistently with our observations this probability will increase with the ionic strength, and decrease when increasing the flow rate (since in laminar conditions, the local velocity increases in the same proportion) or, equivalently, when decreasing the porosity. We can also define $b_{\text {sat }}$ as the maximum value reached by $b$ under given conditions.

For example, for a cylindrical pore throat of radius initially equal to $r_{0}$ but reduced to $r$ by deposited particles along its wall, the adsorption probability writes $p(r)=$ $1-r_{c}^{2} /\left(r-r_{p}\right)^{2}$ [see Fig. 2(b) and Ref. [29] ]. In that case we have $b=1-r^{2} / r_{0}^{2}$, from which we deduce $p(b)$. Remarkably, the simple expression $p_{0}\left(1-b / b_{\text {sat }}\right)$ is a good approximation of $p(b)$ for all pore sizes $r$ greater than $r_{p}$, i.e., as long as the pore is not fully clogged (see Ref. [29] for details and approximated forms of $p_{0}$ and $\left.b_{\text {sat }}\right)$. Looking at the particle deposit as a whole, as a first approximation, it means that the process could still globally be considered as a simple deposition process (see above) with regards to a saturation value, i.e., $b_{\text {sat }}$, depending on $I$.

Extrapolating this result to the more complex pore structure in a bead packing suggests writing the adsorption rate as $\partial b / \partial t=k^{\prime} s\left(1-b / b_{\text {sat }}\right)$, where the factor $s$ appears as the existence of an initial surface layer is required for bulk adsorption. Within our framework, $k^{\prime}$ is a factor including (i) the frequency of attempt of adsorption and (ii) the probability of adsorption of a particle approaching a deposited particle. Therefore, we can write $k^{\prime}=p_{0} / \tau$ with $\tau$ a characteristic time of adsorption attempt, considered as constant here, while $p_{0}$ strongly depends on the ionic strength conditions.

From the above general expression for the bulk adsorption rate $\partial b / \partial t$, and the expression for the surface deposition $s$, we can analytically solve the bulk deposition dynamic 1 as $\left(b / b_{\text {sat }}\right)=1-\exp \left\{\left(k^{\prime} / k\right)\left(1 / b_{\text {sat }}\right)[1-\exp (-k t)-k t]\right\}$. Besides, after rescaling by the surface layer fluorescence, the total deposition $F=S+B=s S_{0}+b B_{0}$ writes $F / S_{0}=$ $s+\beta b$. The predicted trends for $F / S_{0}$ agree well with all our observations [see Fig. 3(a)]: initial deposition limited by the formation of a surface layer $\left(F / S_{0}=1\right)$, and further deposition (if $k^{\prime} \neq 0$ ) decaying to the asymptotic value $1+\beta b_{\text {sat }}$. This is valid up to high ionic strength values $\left(I \sim 10^{0} \mathrm{M}\right)$, above which the particles may easily form large loose clusters, which enhances the clogging probability. To compare our expression for $F / S_{0}$ to the experimental dynamics, $\beta$ is fixed at 6.7. Comparison for $I \in\left[10^{-6} ; 2 \times 10^{-1}\right] \mathrm{M}$, by fitting $k^{\prime}$ (therefore $p_{0}$ ) and $b_{\text {sat }}$, shows modeled dynamics in good agreement with the experimental values [see Fig. 3(a)]. Independently, from 
the model, the values of $p_{0}$ and $b_{\text {sat }}$ are calculated by slight adjustment of $A_{H}$ and $\Phi_{0}$ around their theoretically predicted values [29]. The single value $\tau=3.0 \times 10^{4}$ s then allows us to match experimental and calculated $p_{0}$ and $b_{\text {sat }}$ over 5 decades of ionic strength [see Fig. 3(b)]. We therefore validate our ability to predict the impact of the ionic strength on the bulk deposition probability and saturation.

Overall, the competition between the interparticle interactions and the evolutive hydrodynamics allows us to completely describe the deposition state and rate. The resulting full model can be used to compute the rate of deposition along all regimes, from early single layer surface adsorption to limited multilayers or clogging. The additional approximated model can be used to quickly estimate the existence of a critical saturated state of deposition. Such tools can significantly change the accuracy of contaminant transport predictions. More generally, the mechanism of controlled limited deposition can pave the way to new filtration, cleaning, or sorting systems, with controlled adaptive pore size distributions. As an important next step, confronting these local mechanisms to deeper sections (same dataset) will help answer the fundamental question of depth propagation.

[1] Z. Cai, J. Kim, and M. M. Benjamin, Environ. Sci. Technol. 42, 619 (2008).

[2] R. K. Iler, J. Colloid Interface Sci. 21, 569 (1966).

[3] M. B. Rothberg, Circ. Cardiovasc. Qual. Outcomes 6, 129 (2013).

[4] R. C. Valentine and A. C. Allison, Biochim. Biophys. Acta 34, 10 (1959).

[5] A. G. Matthysse, Front. Plant Sci. 5, 252 (2014).

[6] M. G. Gabridge, Yale J. Biol. Med. 56, 657 (1983).

[7] C. Bianco, J. E. Patiño Higuita, T. Tosco, A. Tiraferri, and R. Sethi, Sci. Rep. 7, 12992 (2017).

[8] J. A. C. Barth et al., Agron. Sustainable Dev. 29, 857 (2009).

[9] S. A. Bradford and M. Bettahar, J. Environ. Qual. 34, 469 (2005).

[10] S. A. Bradford, J. Simunek, and S. L. Walker, Water Resour. Res. 42, W12S12 (2006).
[11] G. Gerber, S. Rodts, P. Aimedieu, P. Faure, and P. Coussot, Phys. Rev. Lett. 120, 148001 (2018).

[12] V. Ramachandran and H. S. Fogler, J. Fluid Mech. 385, 129 (1999).

[13] M. Elimelech, J. Colloid Interface Sci. 146, 337 (1991).

[14] A. Franchi and C. R. O’Melia, Environ. Sci. Technol. 37, 1122 (2003).

[15] Y. Tan, J. T. Gannon, P. Baveye, and M. Alexander, Water Resour. Res. 30, 3243 (1994).

[16] B. Dersoir, M. R. de Saint Vincent, M. Abkarian, and H. Tabuteau, Microfluid. Nanofluid. 19, 953 (2015).

[17] A. Sauret, K. Somszor, E. Villermaux, and E. Dressaire, Phys. Rev. Fluids 3, 104301 (2018).

[18] B. Mustin and B. Stoeber, Microfluid. Nanofluid. 9, 905 (2010).

[19] A. G. Konstandopoulos, Powder Technol. 109, 262 (2000).

[20] N. Ochiai, E. L. Kraft, and J. S. Selker, Water Resour. Res. 42, W12S06 (2006).

[21] A. P. Lehoux, S. Rodts, P. Faure, E. Michel, D. CourtierMurias, and P. Coussot, Phys. Rev. E 94, 053107 (2016).

[22] P. A. Deshpande and D. R. Shonnard, Water Resour. Res. 35, 1619 (1999).

[23] I. Langmuir, J. Am. Chem. Soc. 40, 1361 (1918).

[24] P. R. Johnson and M. Elimelech, Langmuir 11, 801 (1995).

[25] B. Derjaguin and L. Landau, Prog. Surf. Sci. 43, 30 (1993).

[26] E. J. W. Verwey and J. Th. G. Overbeek, J. Phys. Colloid Chem. 51, 631 (1947).

[27] F. Kuhnen, K. Barmettler, S. Bhattacharjee, M. Elimelech, and R. Kretzschmar, J. Colloid Interface Sci. 231, 32 (2000).

[28] R. G. LeBel and D. A. I. Goring, J. Chem. Eng. Data 7, 100 (1962).

[29] See Supplemental Material at http://link.aps.org/ supplemental/10.1103/PhysRevLett.000.000000 for precisions on porous media, working fluid, confocal imaging, interactions quantification, and modeling.

[30] G. K. Batchelor, An Introduction to Fluid Dynamics (Cambridge University Press, Cambridge, England, 2000).

[31] J. H. de Boer, Angew. Chem. 65, 431 (1953).

[32] S. Glasstone, K. J. Laidler, and H. Eyring, The Theory of Rate Processes: The Kinetics of Chemical Reactions, Viscosity, Diffusion and Electrochemical Phenomena. (McGraw-Hill, New York, 1941).

[33] R. F. Probstein, Physicochemical Hydrodynamics: An Introduction (Wiley-Interscience, Hoboken, NJ, 2003). 